\title{
MiR203 Lost Suppressor Genes Function in the Process of Barrett's Esophagus Carcinogenesis Because of High Methylation in Promoter
}

\author{
Liu Tianyu*, Long Xiaoqi \\ Department of Digestion and Gastroenterology, Suining Central Hospital Affiliated to Chongqing Medical University, Suining, China
}

Email address:

58146150@qq.com (Liu Tianyu),drdragon@sina.cn(Long Xiaoqi)

${ }^{*}$ Corresponding author

\section{To cite this article:}

Liu Tianyu, Long Xiaoqi. MiR203 Lost Suppressor Genes Function in the Process of Barrett's Esophagus Carcinogenesis Because of High Methylation in Promoter. Clinical Medicine Research. Vol. 8, No. 1, 2019, pp. 21-26. doi: 10.11648/j.cmr.20190801.14

Received: November 13, 2018; Accepted: January 11, 2019; Published: April 1, 2019

\begin{abstract}
This study was designed to explore the role of MiR203 promoter methylation in the process of Barrett's esophagus carcinogenesis. RT-PCR was used to detect the expression levels of miRNA-203 in Barrett's esophagus, esophageal cancer and normal esophageal mucosa cell lines, before and after the treatment of demethylation. MiR203 promoter methylation levels in these cell lines were measured by Methylation Specific PCR (MSP). Immunohistochemistry was used to test the expression and distribution of K-Ras, a target of miR203, in esophageal cancer, BE and normal esophagus tissues. The following results were found based on the above methods. MiR203 expression levels were reduced obviously in Barrett esophagus and esophageal cancer cells than normal esophageal cells, the difference was statistically significant $(P=0.003)$. After demethylation treatment, miR203 expression levels were significantly increased in Barrett's esophagus and esophageal cancer cells, the differences were statistically significant $(P=0.03)$. MSP results showed that miR203 promoter changed to be low-methylation or non-methylation after demethylation treatment. In conclusion, MiR203 in Barrett's esophagus and esophageal cancer cells reduced expression is related to its Promoter methylation, miR203 promoter methylation throughout the carcinogenesis of Barrett's esophagus, it may become a key molecular biomarker in process of Barrett esophagus cancerous, and may become the prevention and treatment targets of Barrett esophagus carcinogenesis.
\end{abstract}

Keywords: Barrett's Esophagus, miR203, Methylation, Esophagus Carcinogenesis

\section{Introduction}

Barrett's esophagus (BE) was referred to a pathological phenomenon, what occurred in the lower esophagus, that simple columnar epithelium metaplasia replaced the stratified squamous epithelium, whatever with or without intestinal metaplasia, and which accompanied by special types of intestinal metaplasia was domestic grandpa as Esophageal Adenocarcinoma (EAC) of precancerous lesions. The carcinogenesis development process was usually metaplasia-hyperplasia-adenocarcinoma. It was reported that patients with BE risk higher 30-125 times than generals [1]. Therefore, it's very important to find out BE carcinogenesis and its mechanism in early time, and it's certain valuable to determine the molecular marker and therapeutic target.
Epigenetics means the heritable changes in gene expression, but not in DNA sequence. The change way includes DNA methylation, chromatin remodeling, histone modifications and non-coding RNA, etc. DNA methylation is one of the important mechanisms of epigenetics [2], it is closely related to the development of cancer [3, 4]. MicroRNAs (miRNA) is a kind of non-coding small RNAs, with about 22 to 28 nucleotides, it can regulate cell proliferation, differentiation and apoptosis, and it can also regulate the development of cancer by suppressor genes or oncogenes. The epigenetics regulation in miRNA expression plays an important role in the development of cancer, so this study was designed to observe the relationship between miR203 promoter methylation and $\mathrm{BE}$ carcinogenesis.

Main body 


\section{Method}

\subsection{Materials and Methods}

Cell lines were bought from American Type Culture Collection (ATCC), including BE cell lines (B-T、B-T9、 B-T10), esophageal carcinoma cell lines (EC109、TE-10、 SEG-1) and Normal esophageal mucosa cells (NES). MiR203 promoter sequences and transcription start site were predicted by Public Library of Bioinformatics (figure 1). The RT-PCR primer sequences were designed as following sequences, reverse transcription TCAACTGGTGTCGTGGAGTCGGCAATTCAGTTGAGC TAGTGGT, forward primer ACACTCCAGCTGGGGTGAAATGTTTAGGACCA, reverse primer CTCAACTGGTGTCGTGGA. The MSP primer sequences were designed as following sequences, forward primer TGGGTGGTTGTAGTAGGGTAGG, methylation reverse primer ATTAAAAACCACTAAACCCAACG, un-methylation reverse primer CTATTAAAAACCACTAAACCCAACA. All the cell lines were cultured in 1640 culture medium containing 10\% fetal bovine serum. Real-time PCR was used to detect miR203 expression levels above cell lines. $1 \%$ 5-aza-2'-deoxycytidine and $0.1 \%$ Trichostatin A were dissolved in dimethyl sulfoxide as demethylation drugs. MSP was used to detect miR203 promoter methylation levels in certain cell lines. Immunohistochemistry was used to detect $\mathrm{K}-\mathrm{Ras}$ protein expression levels in $\mathrm{BE}$, esophageal cancer and normal esophageal tissues.

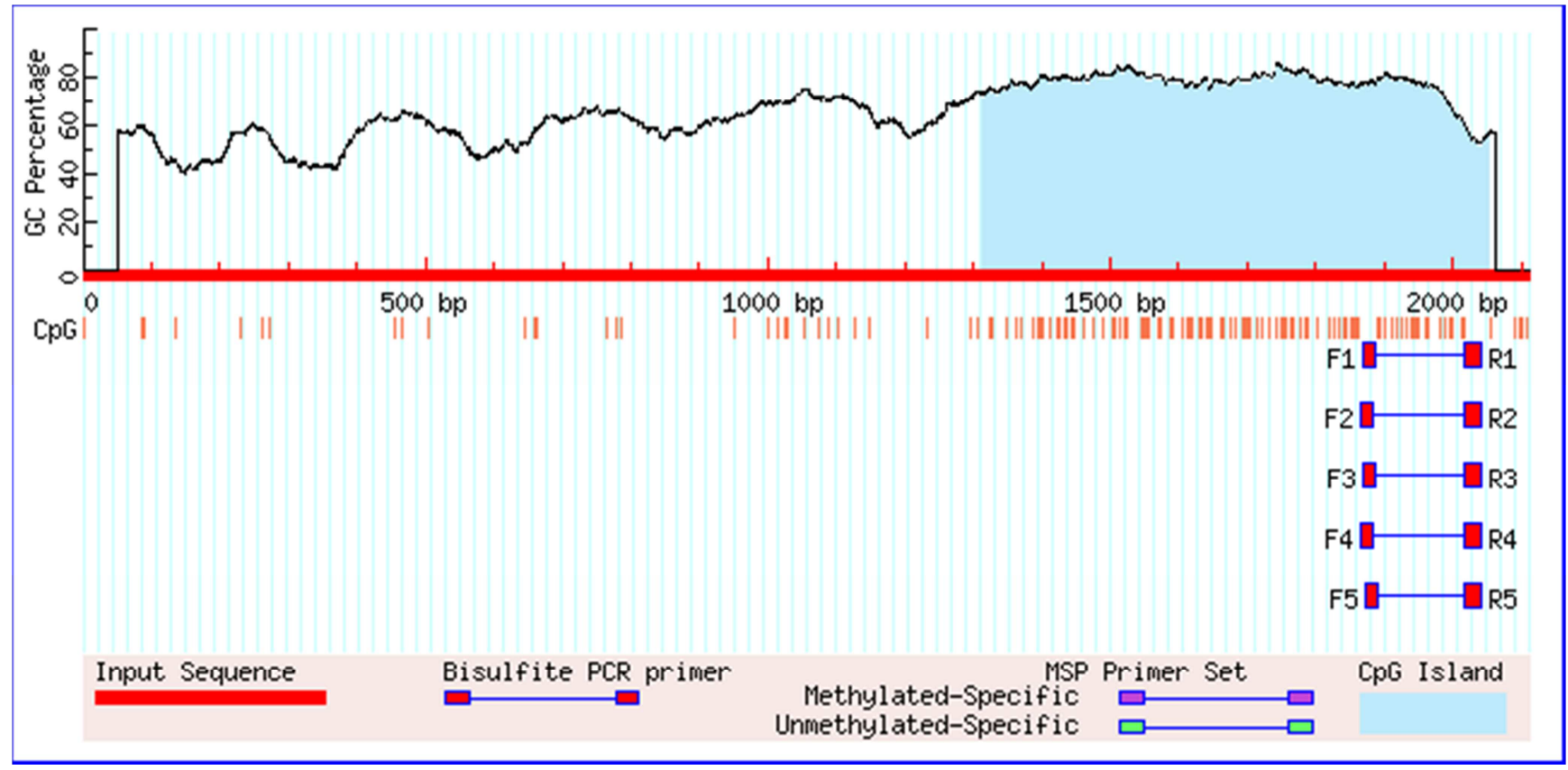

Figure 1. Prediction of miR203 promoter sequences and transcription start site.

\subsection{Statistical Methods}

Spss 22.0 was used as statistical application, LSD test was used to test group comparison. The paired T test was used to detect miR203 expression levels in cell lines before and after demethylation, the differences of miR203 expression levels between diseased tissues and adjacent normal tissues were also detected by paired $\mathrm{T}$ test analysis, Satterthwate t' test was used if variance not neat. $\mathrm{P}<0.05$ for the difference was statistically significant. The methylation rate was calculated of Methylation/Methylation + Un-methylation.

\section{Results}

\subsection{MiR203 Expression Levels in Cell Lines}

RT-PCR tests in cell lines showed that miR203 expressed significantly lower in $\mathrm{BE}$ and esophageal cancer cell lines, especially in B-T and SEG-1 (Figure 2-A). After demethylation treatment, miR203 expression levels in B-T and esophageal cancer cell lines got significantly higher than before, but lower in B-T9 and almost the same in B-T10 (Figure 2-B). The comparison of ascending range showed that it was significantly higher than $\mathrm{BE}$ cell lines after demethylation treatment (Figure 2-C).

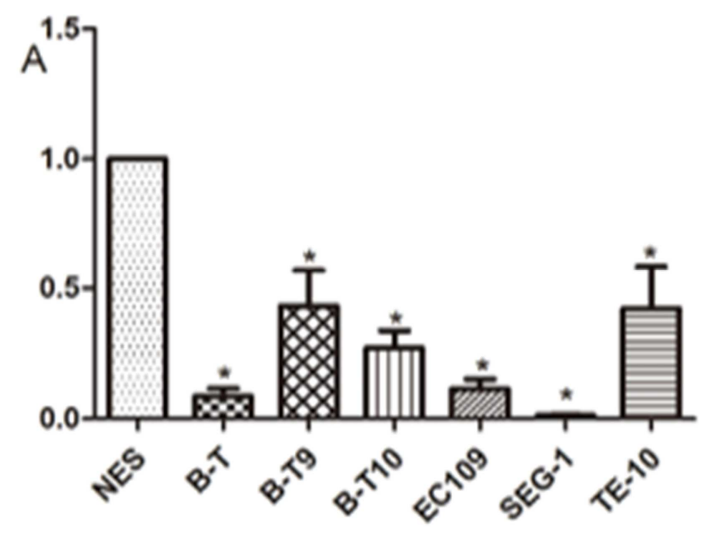




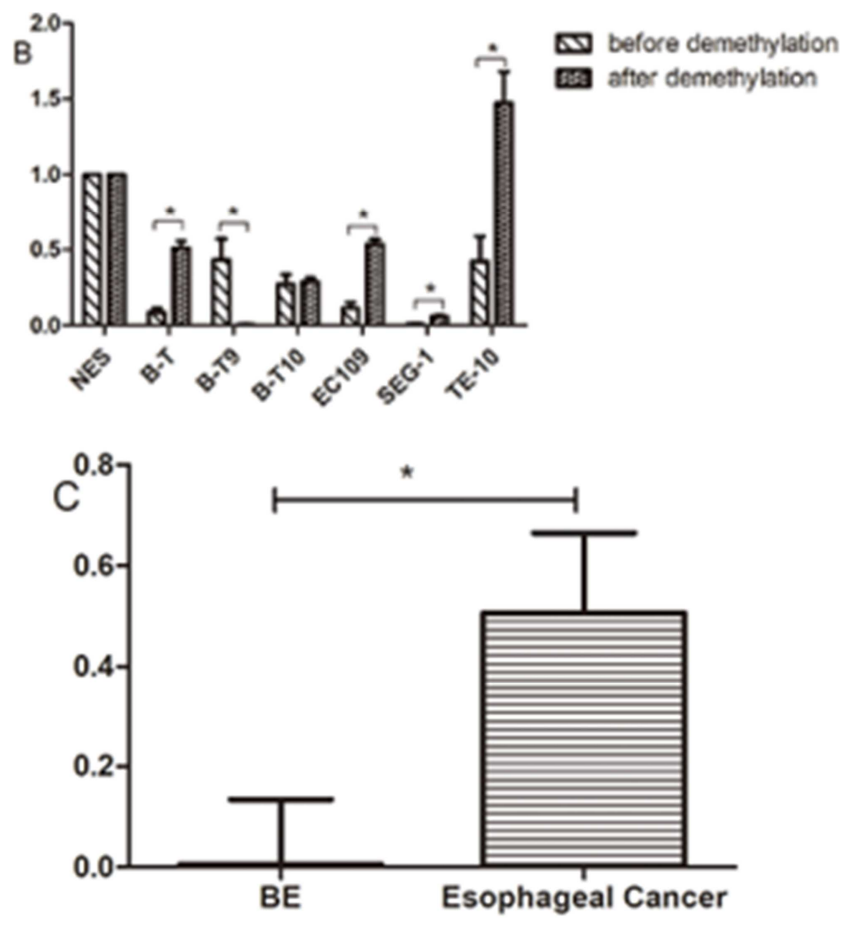

Figure 2. MiR203 expression levels in cell lines. A: each miR203 expression level in cell lines; $B$ : each change of miR203 expression level before and after demethylation; $C$ : change mounts of $B E$ and esophageal cancer cell lines before and after demethylation; *: $P<0.05$.

\subsection{MiR203 Expression Levels in Tissues}

RT-PCR tests in BE and esophageal cancer tissues showed that miR203 expression levels were both lower in them than adjacent normal esophageal tissues, and it was more lower in esophageal cancer tissues than BE (Figure 3).
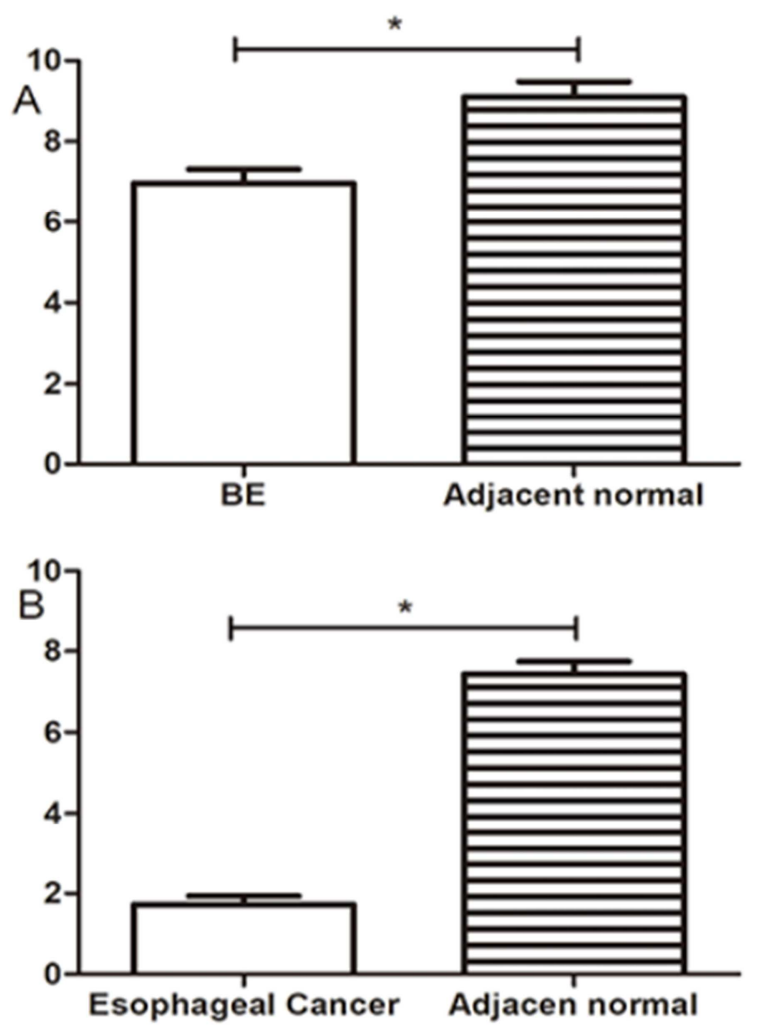

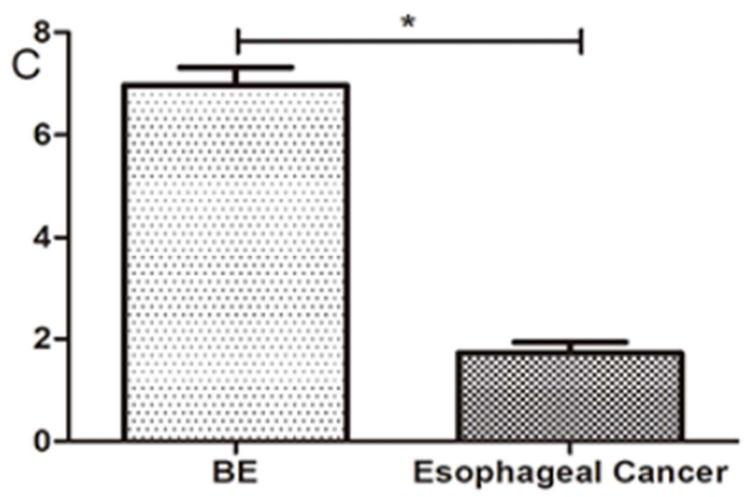

Figure 3. MiR203 expression levels in tissues. A: miR203 expression levels in $B E$ and adjacent normal tissues; $B$ : miR203 expression levels in esophageal cancer and adjacent normal tissues; $C$ : miR203 expression levels in BE and esophageal cancer tissues; *: $P<0.05$.

\subsection{MSP in Cell Lines}

Results of MSP in cell lines showed that methylation and permethylation was found in miR203 promoter in BE and esophageal cancer cell lines, and non-methylation in normal cell lines (Figure 4-A). After demethylation treatment to BE and esophageal cancer cell lines, MSP was used again, the results showed that methylation levels of miR203 promoter in these cell lines changed into low methylation or non-methylation (Figure 4-B). The difference of methylation rate was significantly after demethylation treatment (Figure 4-C).
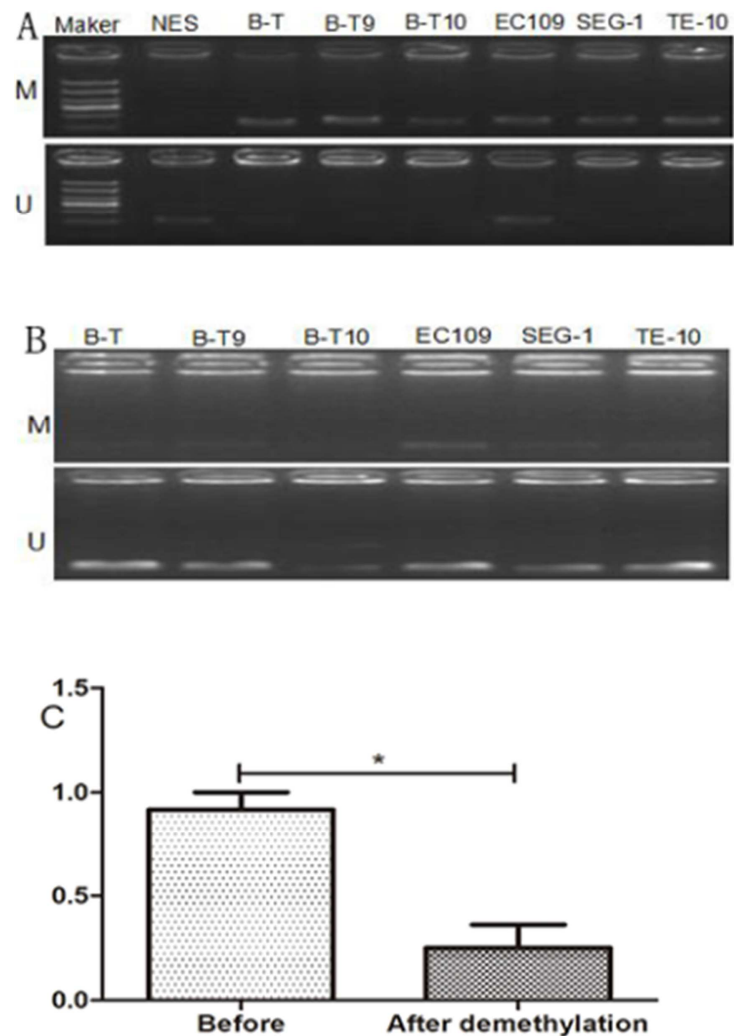

Figure 4. MSP test of miR203 promoter region in cell lines. A: each MSP test of miR203 promoter region in cell lines; B: each MSP test of miR203 promoter region in cell lines after demethylation; $C$ : comparison of methylation rate before and after demethylation; *: $P<0.05$. 


\subsection{MSP in Tissues}

The results of MSP in tissues showed that miR203 promoter methylation were found in BE and esophageal cancer tissues, but low methylation or non-methylation in adjacent normal tissues (Figure 5).
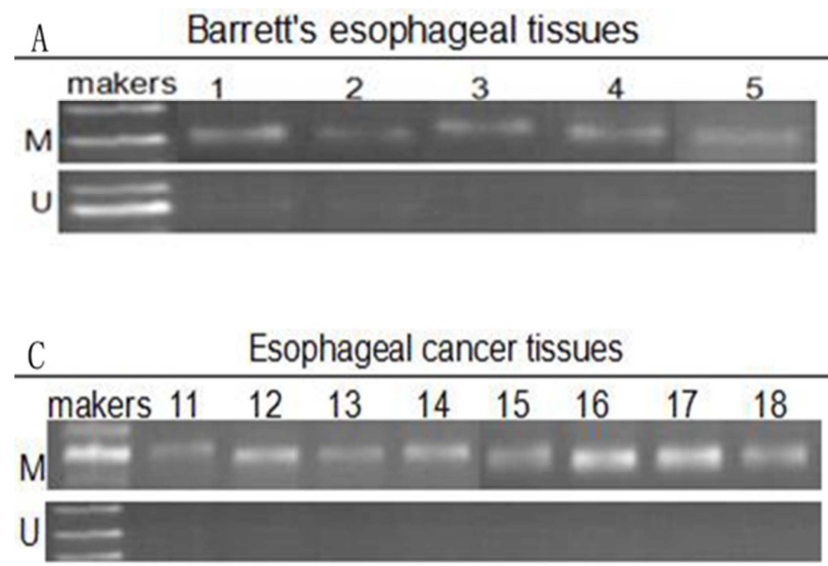

B Barrett's esophageal adjacent tissues
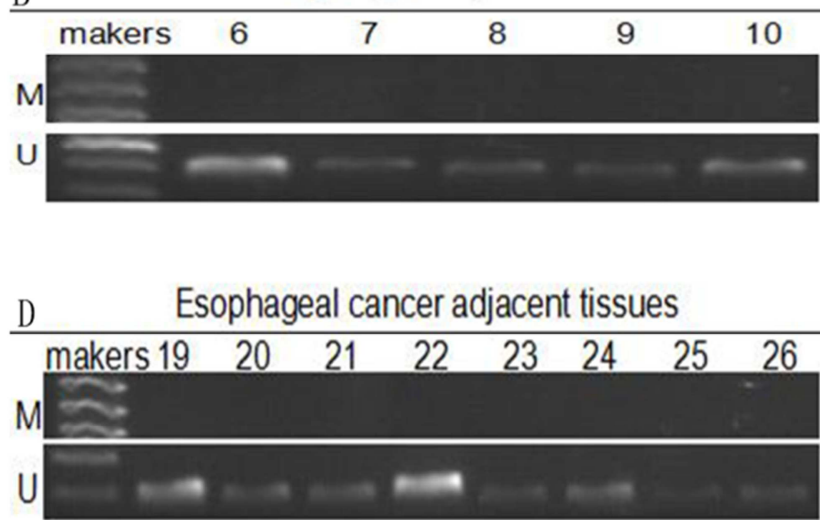

Figure 5. MSP test of miR203 promoter region in tissues. A: each MSP test of miR203 promoter region in BE tissues; B: each MSP test of miR203 promoter region in BE adjacent normal tissues; $C$ : each MSP test of miR203 promoter region in esophageal cancer tissues; D: each MSP test of miR203 promoter region in esophageal cancer adjacent normal tissues; $*$ : $P<0.05$.

\subsection{Protein K-RAS Expression in Tissues}

Protein K-RAS was tested by Immunohistochemistry in BE and esophageal cancer tissues. It expressed higher in BE and esophageal cancer tissues than adjacent normal tissues, and even more higher in esophageal cancer tissues than BE (Figure 6).
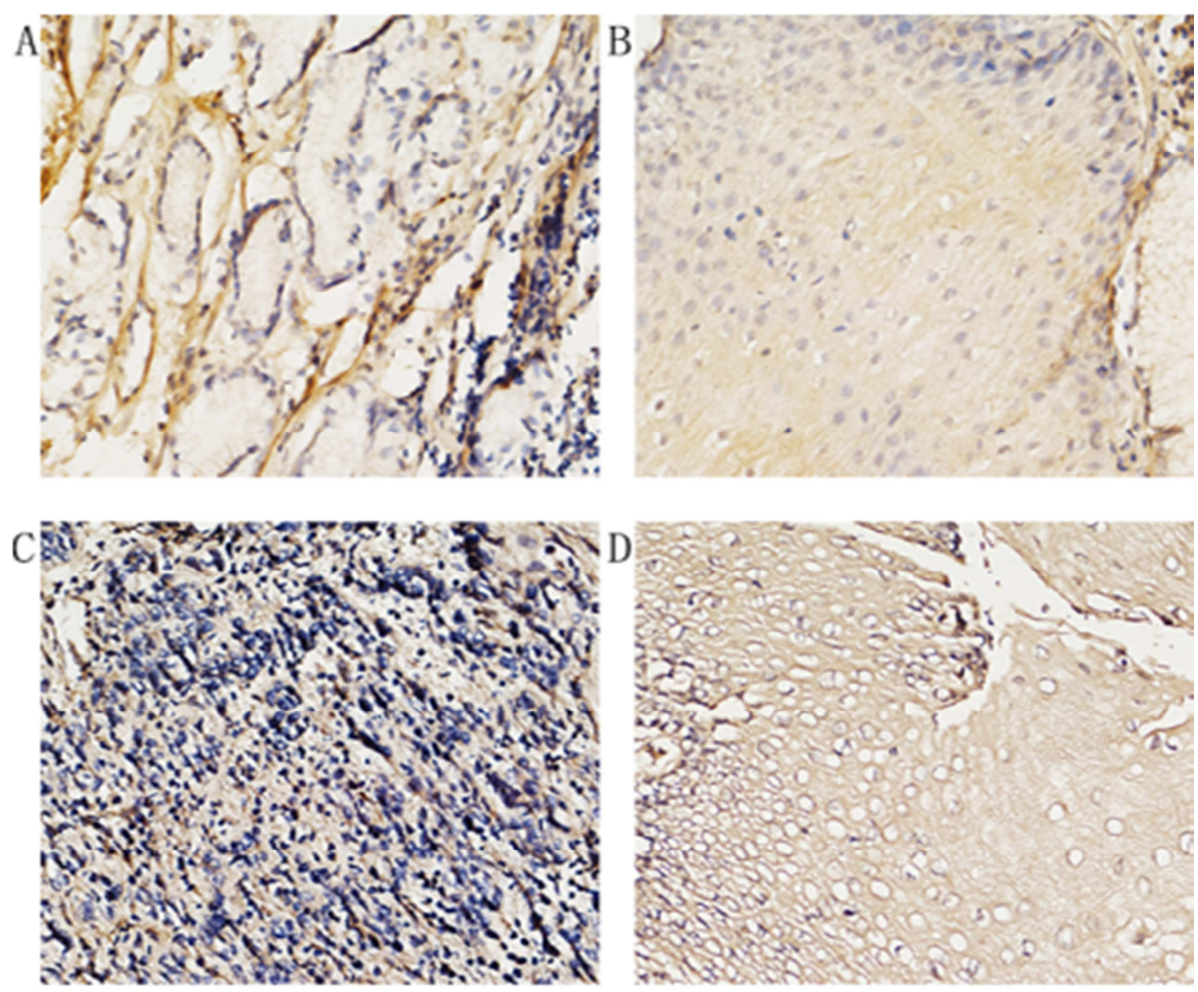

Figure 6. Immunohistochemical test of Protein K-RAS expression in tissues. A: BE tissues; B: BE adjacent tissues; C: esophageal cancer tissues; D: esophageal cancer adjacent tissues.

\section{Discussion}

The diagnosis criteria of $\mathrm{BE}$ contains length and histological lesions, BE was usually divided into Long segmental Barrett's esophagus (LSBE) and short segmental Barrett's esophagus (SSBE) [5]. LSBE had a cancerous rate of $0.5 \% \sim 1 \%$ per year, $8 \% \sim 20 \%$ of adults suffer from SSBE, 
incidence of a disease was higher than LSBE, and about $35 \%$ of the EAC originated from SSBE. Due to the special anatomical site, it was difficult to the difference between $\mathrm{BE}$ and cardiac intestinal change [6]. It was not always perfectly match of squamous-columnar border with esophageal gastric junction by endoscopy [7], and there was lack of identification basis for distinguish of SSBE and gastric mucosa intestinal metaplasia. The aim of this study was to clarify the molecular mechanism of $\mathrm{BE}$ carcinogenesis from epigenetics, and to provide certain theoretical basis for diagnosis and treatment of $\mathrm{BE}$ carcinogenesis. It had been proved of abnormal expression of miRNAs in tumors, and abnormal promoter methylation in these miRNAs [8]. MiRNAs expression level decreased because of high methylation or super methylation in promoter, and then prompted the purpose gene (such as BCL6 [9], CDK6 [10] and MAP3K8 abnormal expression of [11], etc.) expressed abnormally, so that tumorigenesis occurred. Recently several researches examined miR203 expression levels in gastric cancer [12], pancreatic cancer [13], breast cancer [14] and multiple myeloma [15], but little in esophageal cancer and BE, so did its resons.

MiR203 expression levels were detected in cell lines by RT-PCR, the results showed it expressed lower in BE and esophageal cancer cell lines than normal, and it got elevated after demethylation treatment. It prompted that miR203 promoter methylation may exist in these cell lines, and miR203 could re-express or express higher by the way of demethylation. However, cell lines of B-T10 changed little and B-T9 got higher than before, it may be caused by different methylation situation of miR203 promoter or other reasons, such as chromatin remodeling [16] and histone modifications [17]. Then miR203 promoter methylation was detected in cell lines and tissues by MSP, it suggested that methylation or high methylation were found in both BE cell lines and tissues, and it was similar in esophageal cancer cell lines and tissues. After the treatment of demethylation, MSP results showed low methylation or non-methylation in BE and esophageal cancer cell lines, and it was also found in BE and esophageal cancer adjacent normal tissues. The results suggested that low miR203 expression level may be caused by methylation or high methylation in its promoter in $\mathrm{BE}$ and esophageal cancer.

Protein K - RAS mainly expressed in the cell membrane, and thus played an important role in the development process of tumor [18]. Protein K - RAS had been confirmed of abnormal expression in human malignant tumor, especially in digestive system tumors, such as colorectal cancer [19], gastric cancer [20] and liver cancer [21].

Esophageal cancer and precancerous lesions BE were collected as experimental objects, the protein K - RAS was confirmed expressed increased obviously in $\mathrm{BE}$ and esophageal cancer tissues compared to adjacent normal tissues by immunohistochemistry, and especially more obvious in esophageal tissues. It suggested that protein K - RAS expressed abnormally in precancerous lesions $\mathrm{BE}$ and esophageal cancer, as the same as other human malignant tumors. Combined with the corresponding miR203 expression in tissues and cells, it may be suggested that protein K - RAS expression in $\mathrm{BE}$ and esophageal cancer may be affected by abnormal miR203 expression level, miR203 may be one of the protein K - RAS expression regulation factors, and it was matched with results in ovarian cancer [22].

\section{Conclusion}

This study found that the miR203 expression level was associated with BE carcinogenesis by measuring it in cell lines and tissues, and furthermore it was relevant to methylation or high methylation in its promoter region. So it could illustrate that miR203 expression and its methylation could be one of the evaluable indicators in $\mathrm{BE}$ carcinogenesis, and could provide certain theoretical basis for $\mathrm{BE}$ carcinogenesis prevention and treatment. Based on this research, miR203 could be seemed as a new bio-marker in the diagnosis or process of following up in patients with $\mathrm{BE}$.

\section{Acknowledgements}

This study was supported by Suining science and technology project fund $(2015 \mathrm{~s} 28)$. I would appreciate to Xiaoqi-Long, the director of endoscopy room of Suining central hospital affiliated to Chongqing medical university, to collect tissues, and appreciate to the staff of pathology in Suining central hospital affiliated to Chongqing medical university to help me to accomplish pathological detect.

\section{References}

[1] Feber A, Xi L, Luketich J D, et a1. MicroRNA expression profiles of esophageal cancer [J]. Thorac Cardiovasc Surg, 2008, 135 (2):255-260.

[2] Szigeti KA, Galamb O, Kalmár A, et al. Role and alterations of DNA methylation during the aging and cancer [J]. Orv Hetil. 2018, 159 (1):3-15.

[3] Rahertson KD. DNA methylation and human disease [J]. Nat RevGe net, 2005, 6 (8):597-610.

[4] Lü L, Liu T, Gao J, et al. Aberrant methylation of microRNA-193b in human Barrett's esophagus and esophageal adenocarcinoma [J]. Mol Med Rep. 2016, 14 (1):283-8.

[5] Belghazi K1, van Vilsteren FGI1, Weusten BLAM2, et a1. Long-term follow-up results of stepwise radical endoscopic resection for Barrett's esophagus with early neoplasia [J]. Gastrointest Endosc, 2017, 4 (25): 31820-318255.

[6] Zhang W, Wang DH. Origins of Metaplasia in Barrett's Esophagus: Is this an Esophageal Stem or Progenitor Cell Disease? [J]. Dig Dis Sci. 2018, 63 (8):2005-2012.

[7] Rajendra S, Sharma P. Barrett Esophagus and Intramucosal Esophageal Adenocarcinoma [J]. Hematol Oncol Clin North Am. 201731 (3):409-426.

[8] Noguchi S, Mori T, Nakagawa T, et a1. DNA methylation contributes toward silencing of antioncogenic microRNA-203 in human and canine melanoma cells [J]. Melanoma Res. 2015, 25 (5):390-398. 
[9] Yang J, Wang S, Wang F, et a1. Downregulation of miR-10b promotes osteoblast differentiation through targeting Bcl6 [J]. Int J Mol Med. 2017, 39 (6):1605-1612.

[10] Trohatou O, Zagoura D, Orfanos NK, et a1. miR-26a mediates adipogenesis of amniotic fluid mesenchymal stem/stromal cells via PTEN, Cyclin E1, and CDK6 [J]. Stem Cells Dev. 2017, 26 (7):482-494.

[11] Liu F, Chen N, Xiao R, et a1. MiR-144-3p serves as a tumor suppressor for renal cell carcinoma and inhibits its invasion and metastasis by targeting MAP3K8. Biochem Biophys Res Commun [J]. 2016, 480 (1):87-93.

[12] Hao Q, Lu X, Liu N, et al. Posttranscriptional deregulation of Src due to aberrant miR34a and miR203 contributes to gastric cancer development [J]. BMB Rep. 2013, 46 (6):316-21.

[13] Xu D, Wang Q, An Y, et al. MiR-203 regulates the proliferation, apoptosis and cell cycle progression of pancreatic cancer cells by targeting Surviving [J]. Mol Med Rep. 2013; 8 (2):379-84.

[14] DeCastro AJ, Dunphy KA, Hutchinson J, et al. MiR203 mediates subversion of stem cell properties during mammary epithelial differentiation via repression of $\triangle \mathrm{NP} 63 \alpha$ and promotes mesenchymal-to-epithelial transition [J]. Cell Death Dis. 2013 28; 4:e514.

[15] Wong KY, Liang R, So CC, et al. Epigenetic silencing of MIR203 in multiple myeloma [J]. Br J Haematol. 2011; 154 (5):569-78.
[16] Qu H, Xu W, Huang Y, Yang S, et al. Circulating miRNAs: Promising Biomarkers of Human Cancer [J]. Asian Pacific journal of cancer prevention: APJCP 2011, 12 (5):1117-25.

[17] Lee S, Jung JW, Park SB, et al. Histone deacetylase regulates high mobility group A2-targeting microRNAs in human cord blood-derived multipotent stem cell aging [J].. Cell Mol Life Sci. 2011 Jan; 68 (2):325-36.

[18] Nussinov R, Wang G, Tsai CJ, et al. Calmodulin and PI3K Signaling in KRAS Cancers [J]. Trends Cancer. 2017, 3 (3):214-224.

[19] Markman B, Javier Ramos F, Capdevila J, et al. EGFR and KRAS in colorectal cancer [J]. Adv Clin Chem, 2010; 51:71-119.

[20] Sug Hyung Lee, Jong Woo Lee, et al. Young Hwa Soung, et al. BRAF and KRAS mutations in stomach cancer [J]. Oncogene 2003, 22, 6942-6945.

[21] S. Stremitzer, J. Stift, B. Gruenberger, et al. KRAS status and outcome of liver resection after neoadjuvant chemotherapy including bevacizumab [J]. British Journal of Surgery, 2012, 99 (11), 1575-1582.

[22] Nakano H, Yamada Y, Miyazawa T, et al. Gain-of-function microRNA screens identify miR-193a regulating proliferation and apoptosis in epithelial ovarian cancer cells [J]. Int J Oncol. 2013, 42 (6):1875-82. 